\title{
Optical-electronic sensor of spacecraft heat protection ablation
}

\author{
G. Hornostaev \\ I. Frantsevich Institute for Problems of Materials Science, NAS of Ukraine \\ 3, Krzhizhanovsky str., 03142 Kyiv, Ukraine \\ E-mail: cosmos@ipms.kiev.ua; phone: (38-044) 424-24-44, fax: (38-044)424-21-31
}

\begin{abstract}
Block diagram and results of tests on gas-dynamic stands are represented to illustrate operation of the continuous ablation sensor, in which color light guides with a diameter of $1 \mathrm{~mm}$ are used. It is shown that the application of compensating light filter in the colorless channel makes it possible to create the meter invariant to external radiant fluxes applied to the heat protection covering surface.
\end{abstract}

Keywords: heat protection, ablation, light guide, sensor.

Manuscript received 26.02.08; accepted for publication 20.06.08; published online 15.09.08.

\section{Introduction}

With the aim to optimize both the thickness and the weight of heat protection coatings covering the being got down spacecraft, the latter is supplied with sensors of ablation of these coatings.

In the analog circuit of sensor measurements [1, 2], the light transmission of two light guides: color (where the light transmission depends on the guide length) and colorless are compared (Fig. 1). The light transmission is measured by two photoresistors CФ3-1. An advantage of the bridge sensor is the independence of the voltage of midpoint from both the external illumination and the temperature of the heat protection surface.

\section{Substantiation of the principle of measurement}

The results obtained earlier [3] show that both color glass and photodetector are wide-band. Combined action of three factors: the spectral energy distribution of the emission, the spectral sensitivity $S(\lambda)$ of detector and

$W(\lambda) d \lambda=\frac{4.991 \cdot 10^{-15}}{\lambda^{5}}\left(e^{\frac{1.43844}{\lambda T}}-1\right)^{-1}$

color light guide transmission $\tau(\lambda)$ determines the effective illumination $J$ (Fig. 2):

$J=\int_{\lambda_{1}}^{\lambda_{2}} W(\lambda) \tau(\lambda) S(\lambda) d(\lambda)$.
Here $\lambda_{1}$ and $\lambda_{2}$ are minimum and maximum wavelengths that correspond to the range of the detector sensitivity. A close agreement of spectral characteristics of color light guide and detector occurs. This makes it possible to do measurements in the narrow fixed spectral range.

\section{Numerical calculations of the effective illumination}

Calculation by the numerical integration method is made for the photoresistor CФ3-1 as well as the color light guides with various lengths of neutral grey glass NG-3 (Fig. 3). The color temperature of the emitter is of 1273$2273 \mathrm{~K}$. Step of the integration $d \lambda=25 \mathrm{~nm}$, the spectral range $\lambda=675-1200 \mathrm{~nm}$, the single section of light guide is of $5 \mathrm{~mm}$.

With a change in the length of color light guide to the value of $\Lambda_{r}$, the photoresistivity changes by $e$ times. The estimation of the length $\Lambda_{r}$ is accomplished according to the amplitude characteristic of the photodetector

$\lg R=h-c \lg J$,

where: $R$ is the value of photoresistance located under the color light guide, $c=\Lambda_{t} / \Lambda_{r}, h=$ const, $\Lambda_{t}$ is determined from Fig. 3. With a change in the length of color light guide to the value $\Lambda_{t}$, its light transmission $\tau$ changes by $e$ times.

With the change of the temperature of emitter the constant value $\Lambda_{t}$ remains. A constant relationship between the illumination in the color channel of the specific length and in the colorless channel (with the zero length of the color light guide) also remains. 


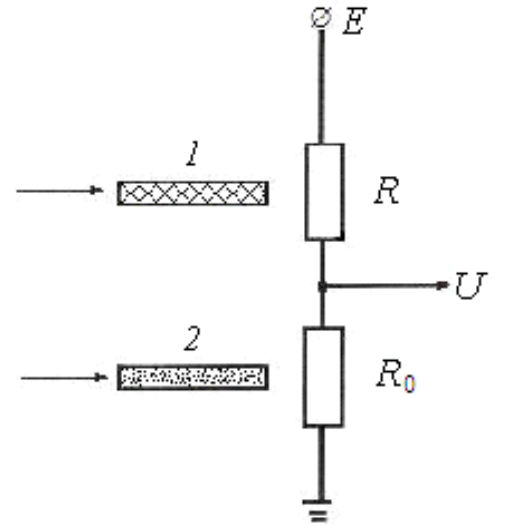

Fig. 1. Bridge scheme of the sensor: 1,2- color and colorless light guides; $R_{0}$ - photoresistance, which is located under the colorless light guide.

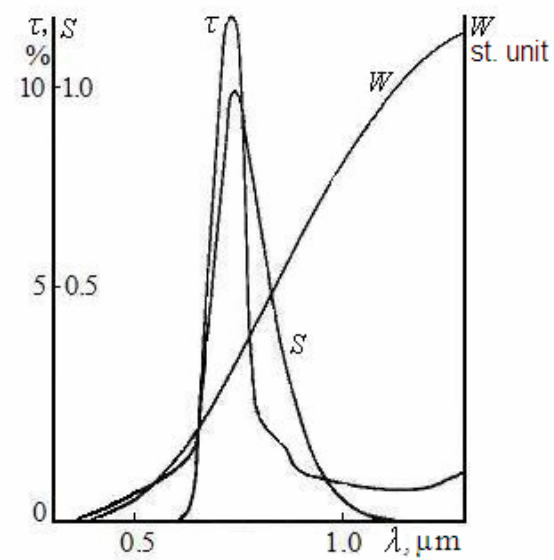

Fig. 2. Factors that influence on the effective illumination: $\tau$ is the transmission of glass NG-3 with the thickness $10 \mathrm{~mm}, W-$ radiant energy with the color temperature $1750{ }^{\circ} \mathrm{C}, S$ - relative light sensitivity of CФ3-1.

\section{Construction of photodetector part}

For the bridge to be invariant to the illumination, it is necessary to establish compensator (light filter) between the colorless light guide and photodetector. This ensures the light transmission equal to the light transmission of the color light guide of the standard length. Approximately, it is possible to accept

$U=\frac{1}{2} E$,

$$
L=L_{2}+\frac{1}{2}\left(L_{1}-L_{2}\right) \text {. }
$$

Here: $L_{1}, L_{2}$ are the initial and finite lengths of the color light guide; $E$ - supply voltage; $U$ - voltage of the midpoint of potentiometer; $L$ - certain standard length of the light guide: $\left(L_{1}>L>L_{2}\right)$. The compensator is divided by two parts: the filter of coarse adjustment (of the glass NG-2 with the length $10 \mathrm{~mm}$ ) and glass post with the black segment. It is possible to change the value of the photo resistance $R_{0}$ located under the colorless light guide (Table) by means of detector rotation.
Table. Data on flow control with the aid of the compensator of the fine adjustment.

\begin{tabular}{|c|c|c|}
\hline $\begin{array}{c}\text { Mark of the glass } \\
\text { post }\end{array}$ & $\begin{array}{c}\text { Painting the side } \\
\text { surface }\end{array}$ & $R_{0}, \mathrm{KOhm}$ \\
\hline $\mathrm{BC}$ & White & $7-500$ \\
\hline $\mathrm{BC}$ & Black & $14-2000$ \\
\hline $\mathrm{NC}$ & White & $88-1500$ \\
\hline $\mathrm{NC}$ & Black & $62-1500$ \\
\hline
\end{tabular}

\section{Stand tests}

To check the correctness of calculations of the parameter $\Lambda_{r}$, the measurement of Teflon-4 ablation with the aid of the color light guides of the glass NG-3 was carried out. The inspection of the value of removal is achieved with the aid of the filming. Tests were performed using two gas-dynamic stands (IPMS NAS of Ukraine, Kyiv and SRI TP, Moscow).

Results of measurements (Fig. 4) showed that the composition of medium and its thermodynamic parameters do not influence on the value of the parameter $\Lambda_{r}=3.85 \mathrm{~mm}$. This value corresponds to the results of calculation illustrated in Fig. 3.

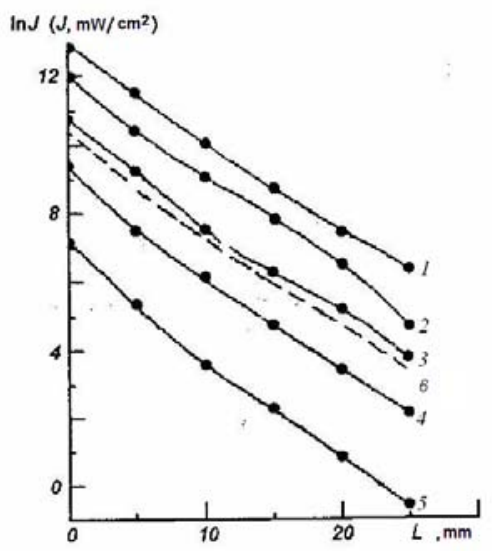

Fig. 3. Effective illumination of photoresistor CФ3-1 calculated in dependence on the length $L$ of the light guide made of the glass NG-3 at various color temperatures $T$ of the illuminator: $1-\Lambda_{r}=3.975 \mathrm{~mm}, T=2273 \mathrm{~K} ; 2-\Lambda_{r}=3.65 \mathrm{~mm}$, $T=2023 \mathrm{~K} ; 3-\Lambda_{r}=4.40 \mathrm{~mm}, T=1773 \mathrm{~K} ; 4-\Lambda_{r}=3.78 \mathrm{~mm}$, $T=1523 \mathrm{~K} ; 5-\Lambda_{r}=3.90 \mathrm{~mm}, T=1273 \mathrm{~K} ; 6-$ average values.

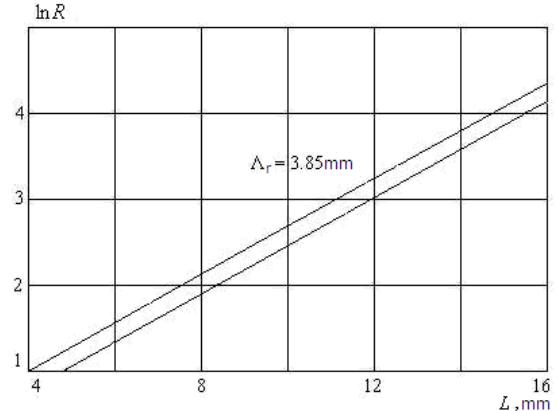

Fig. 4. Change in the value of the photoresistance located under the color light guide with the diameter $1 \mathrm{~mm}$ and the length $15 \mathrm{~mm}$ made of glass NG-3 in the process of Teflon-4 ablation on two gas-dynamic stands ( $L$ is the instantaneous value of the color light guide length): $1-q=12 \mathrm{MW} / \mathrm{m}^{2}, P=2.3 \cdot 10^{5} \mathrm{~Pa}, T=$ $2600 \mathrm{~K} ; 2-q=16 \mathrm{MW} / \mathrm{m}^{2} ; P=5.5 \cdot 10^{5} \mathrm{~Pa}, T=5500 \mathrm{~K}$.

\section{(C) 2008, V. Lashkaryov Institute of Semiconductor Physics, National Academy of Sciences of Ukraine}




\section{Conclusions}

1. It is proved by both the calculation way and the experiment on two gas-dynamic stands that the constant of the length of color light guide is stable under varied illumination conditions $\left(\Lambda_{r}=3.85 \mathrm{~mm}\right)$. It is necessary to introduce compensating light filter into the colorless channel. Its transmission (in the maximum of the band of the spectral sensitivity of detector) must be close to the transmission of the color light guide which shortened to half the thickness of the removed layer.

2. The results of measurements of the removal of heat protection material Teflon- 4 obtained by the lightguide sensor and filming coincide with the accuracy $\leq 10 \%$.

\section{References}

1. G.F. Hornostaev, Fiber-optic sensors and the prospect for their use in the space program of Ukraine // Kosmichna nauka i tekhnologiya 2(3/4), p. 88-94 (1996) (in Russian).

2. G.F. Hornostaev, V.V. Pasichny and G.V. Tkachenko, Method of measurement by radiant component of heat flux on the surface of ceramic heat protection // Kosmichna nauka i tekhnologiya 12(2/3), p. 98-102 (2006) (in Russian).

3. G.F. Hornostaev, V.V. Pasichny and G.V. Tkachenko, Methods of the control of operating characteristics of heat protection by the fiber-optic sensors // Kosmichna nauka i tekhnologiya 13(3), p. 12-18 (2007) (in Russian). 